\section{Fatores ambientais associados à diarréia infantil em áreas de assentamento subnormal em Juiz de Fora, Minas Gerais}

\section{Childhood diarrhea-related to environmental factors in subnormal settlements in Juiz de Fora, Minas Gerais}

Júlio César Teixeira 1

Léo Heller 2
Abstract

Objectives: to determine diarrhea prevalence and to identify factors associated to the condition in children living in subnormal settlement areas.

Methods: a cross-sectional population-based study was used. The sample studied totaled 655 children. Data collection was performed through home interviews with the mother or caretaker. Logistic regression models were used to identify diarrhea associated factors.

Results: diarrhea prevalence was of $17.5 \%$. Condition associated factors included child hospitalization during the first month of life; children looked after by others and not the mother; higher education level of the caretaker and longer residency period, as protection factors and as individual risk factors associated diseases and young age and as factors for collective risk, mine water consumption; sewage disposal on the streets or in the yard; inadequate garbage disposal and flies.

Conclusions: to fight diarrhea special care should be given to health and nutrition of children under five years old, including adequate and quality environmental sanitation services.

Key words Diarrhea, infantile, Child welfare, Sanitation, Water, Sewage

\section{Resumo}

Objetivos: caracterizar a prevalência de diarréia e identificar os fatores associados à essa doença em crianças residentes em áreas de assentamento subnormal.

Métodos: utilizou-se um delineamento transversal de base populacional. A amostra estudada totalizou 655 crianças. A coleta de dados foi feita mediante entrevistas domiciliares com a mãe ou com o responsável pela criança. Foram usados modelos de regressão logística para identificar fatores associados à diarréia.

Resultados: a prevalência da diarréia foi 17,5\%. Os fatores associados à doença incluíram internação da criança no primeiro mês de vida; ser cuidado por terceiros, que não a mãe; maior nível de escolaridade do responsável; e maior tempo de residência, como fatores de proteção. Como fatores de risco individuais, a existência de outra doença; e baixa idade. Como fatores de risco coletivos, o consumo de água de mina; a disposição dos esgotos na rua ou no terreno; o acondicionamento inadequado do lixo; e presença de moscas. Conclusões: para o combate à diarréia sugere-se atenção especial à saúde e à alimentação de crianças com idade abaixo de cinco anos, incluindo adequadas cobertura e qualidade nos serviços de saneamento ambiental.

Palavras-chave Diarréia infantil, Bem-estar da criança, Saneamento, Água, Águas residuais 


\section{Introdução}

A diarréia infantil é um importante problema de saúde infantil em todo o mundo, principalmente nos países em desenvolvimento como o Brasil, devido, entre outros fatores, a inexistência ou precariedade dos serviços de saneamento. Estima-se que a mortalidade a ela atribuída atinja a 1,5 milhão de crianças menores de cinco anos, por ano, no mundo. ${ }^{1}$

A prevalência da doença em algumas regiões do Brasil, como a Nordeste, chega a 15,4\% e em outras, como a Sul, a $5,9 \%$, portanto é uma patologia de impacto considerável. ${ }^{2}$

Os fatores de risco associados à diarréia podem ser explicados dentro de um modelo multicausal que inclui uma extensa quantidade de fatores socioeconômicos, políticos, demográficos, sanitários, ambientais e culturais inter-relacionados. 3

"As enfermidades associadas à deficiência ou inexistência de saneamento ambiental e a conseqüente melhoria da saúde devido à implantação de tais medidas têm sido objeto de discussão em diversos estudos. Entre essas doenças, a diarréia e as doenças parasitárias, em particular, as verminoses, e mais recentemente, a desnutrição, têm merecido atenção de estudiosos e das autoridades sanitárias em todo o mundo". (Moraes; 1997: 282). 4

Entretanto, a prevalência dessas doenças em áreas de assentamento subnormal - áreas de invasão - é pouco ou nada estudada em nosso país, considerando-se períodos recentes.

Segundo dados da Prefeitura de Juiz de Fora, Minas Gerais, 5 relativos ao ano de 2000, cerca de 78 áreas, alvos de invasões, abrigavam aproximadamente 32 mil pessoas em 8,4 mil moradias. Isso significa que pelo menos $7,0 \%$ da população de Juiz de Fora (456 mil habitantes) viviam em situação precária. Dados do Censo de 1991 da Fundação Instituto Brasileiro de Geografia e Estatística (IBGE) 6 mostravam que o número de residentes em assentamentos subnormais no município era de 19.135 pessoas. Segundo as fontes acima, no período 1991 a 2000, o número de pessoas que vive em áreas de assentamento subnormal no município - áreas de invasão - cresceu 3,1 vezes mais do que a população de toda a cidade, respectivamente, $5,9 \%$ e $1,9 \%$ ao ano.

As áreas de assentamento subnormal caracterizam-se pela presença de barracos erguidos com alvenaria ou com pedaços de madeira, plástico e papelão, com precária infra-estrutura básica como, por exemplo, abastecimento de água, esgotamento sanitário, limpeza urbana e energia elétrica. Nessas moradias, muitas vezes falta banheiro, e o chão é normalmente de terra batida, o que, somado à baixa renda familiar, fornece uma dimensão das dificuldades que cada um dos cidadãos residentes nessas áreas enfrenta a cada dia.

Como em todo o país, a maioria dos moradores de áreas de habitação subnormal é desempregada, subempregada ou migrante de cidades menores, que vêem, em cidades de grande ou médio porte, a chance de uma vida melhor. Assim, frente à falta de emprego e de renda, o problema de ocupação de assentamentos subnormais vem se agravando nos últimos anos, expondo milhões de brasileiros a condições de vida precárias, principalmente em relação à habitação, ao saneamento ambiental e às condições de higiene.

O objetivo deste estudo foi caracterizar a morbidade por diarréia e identificar os fatores a ela associados em crianças, com idade entre um ano completo e cinco anos incompletos, residentes em áreas de assentamento subnormal.

\section{Métodos}

O presente estudo consistiu de uma pesquisa transversal, realizada no período de maio a julho de 2002 na cidade de Juiz de Fora, Minas Gerais, Brasil.

A seleção das áreas de estudo foi realizada após contato com órgãos da Prefeitura de Juiz de Fora, a saber, Companhia de Saneamento Municipal S.A. (CESAMA) e Empresa Regional de Habitação de Juiz de Fora S.A. (EMCASA), e Organizações NãoGovernamentais, como a Pastoral da Criança da Arquidiocese de Juiz de Fora e o Centro de Defesa dos Direitos Humanos (CDDH). Considerando as exposições a estudar, 29 assentamentos, com aproximadamente 2.700 moradias e população estimada em 12.000 habitantes, foram selecionados, de forma não aleatória, para compor a amostra de estudo.

A escolha das áreas de estudo de forma não aleatória teve como objetivo a obtenção de diferentes cenários de saneamento ambiental na amostra, visando o seu confrontamento.

O tamanho da amostra calculada foi de 650 crianças, com idade entre um ano completo e cinco anos incompletos, residentes em áreas de assentamento subnormal. No dimensionamento, utilizou-se o programa Epi-info 6.04, adotando-se: alfa=5\%; beta $=20 \%$; razão não-exposto/exposto de $1: 1$; prevalência esperada entre os expostos $=36,4 \%, 7 \mathrm{e}$ prevalência esperada entre os não-expostos $=7,2 \% .7$

A amostra selecionada correspondeu aproxi- 
madamente à totalidade das moradias com crianças nos 29 assentamentos. Nesses, em cada habitação visitada, onde existiam crianças na faixa etária de interesse, uma criança foi sorteada para ser objeto de entrevista, com a verificação da ocorrência ou não de diarréia nas 72 horas que antecederam a entrevista.

Foram aplicados 655 protocolos de entrevista nos 29 assentamentos objeto de estudo. As entrevistas foram realizadas por equipe treinada especificamente para este fim, recrutada entre entrevistadores que trabalharam no Censo 2000 na cidade de Juiz de Fora. A coleta de dados consistiu em entrevista domiciliar com a mãe ou com o responsável pela criança. Na entrevista, foram utilizados questionários estruturados.

Doentes: crianças selecionadas, com idade entre um ano completo e cinco anos incompletos, residentes na área geográfica de estudo, que tenham apresentado diarréia nas 72 horas que antecederam a realização da entrevista, isso é, aumento na freqüência de evacuações e/ou redução na consistência das fezes.

Não-doentes: crianças selecionadas, com idade entre um ano completo e cinco anos incompletos, residentes na área geográfica de estudo, que não tenham apresentado diarréia nas 72 horas que antecederam a realização da entrevista, isso é, sem alterações na frequiência de evacuações e na consistência das fezes.

Fez-se inicialmente uma análise exploratória dos dados, por meio de tabelas de freqüências simples, além da análise univariada com medidas de associação e testes de significância. Posteriormente, aplicou-se a técnica de regressão logística para a análise multivariada dos dados por meio de um processo evolutivo, em etapas seqüenciais, de forma a permitir a eliminação progressiva das exposições não associadas à doença. Tal processo envolveu as seguintes atividades:

a) Seleção preliminar de 31 variáveis, divididas em oito subgrupos, com significância inferior a 0,25 $(p<0,25)$ para integrar o modelo inicial, conforme a seguir:

- Estrutura familiar e estado nutricional da criança: 10 variáveis;

- Nível socioeconômico: sete variáveis;

- Hábitos higiênicos: duas variáveis;

- Abastecimento de água: duas variáveis;

- Esgotamento sanitário: três variáveis;

- Manejo de resíduos sólidos: quatro variáveis;

- Drenagem pluvial: duas variáveis;

- Presença de vetores: uma variável. b) Construção de modelos por subgrupos, com eliminação de variáveis que apresentassem significância inferior a $0,15(p<0,15)$;

c) Construção do modelo final com as variáveis remanescentes em cada um dos oito subgrupos, com a manutenção de variáveis que apresentassem significância inferior a $0,05(p<0,05)$.

A análise dos dados foi desenvolvida por meio do software Statistical Package for the Social Sciences (SPSS), versão 10.0.

O Comitê de Ética em Pesquisas (COEP) da Universidade Federal de Minas Gerais (UFMG) aprovou o presente estudo, estando os procedimentos de acordo com os padrões éticos do comitê responsável por experimentos com humanos e de acordo com a Declaração de Helsinki de 1964, reformulada em 2000 e, ainda, de acordo com a Resolução 196/96 do Conselho Nacional de Saúde (CNS).

\section{Resultados}

De um total de 655 crianças, $348(53,1 \%)$ meninos e $307(46,9 \%)$ meninas com mediana de idade de três anos, $115(17,5 \%)$ tiveram diarréia no prazo de até 72 horas que antecederam a entrevista domiciliar. $\mathrm{Na}$ Tabela 1 são mostradas as principais características apresentadas pelos doentes, destacando-se que em cerca de $80 \%$ dos casos não há referência da ida ao serviço médico, a despeito de $46 \%$ ainda apresentarem diarréia no momento da entrevista.

A Tabela 2 mostra as variáveis que permaneceram associadas à diarréia após o ajuste dos fatores de confusão. As variáveis dos subgrupos hábitos higiênicos e drenagem pluvial não apresentaram associação com a diarréia. Cinco variáveis do subgrupo estrutura familiar e estado nutricional da criança e uma variável de cada um dos subgrupos nível socioeconômico, abastecimento de água, esgotamento sanitário, manejo de resíduos sólidos e presença de vetores demonstraram associação estatística com a diarréia. 
Tabela 1

Caracterização da morbidade por diarréia em crianças com idade entre um ano completo e cinco anos incompletos.

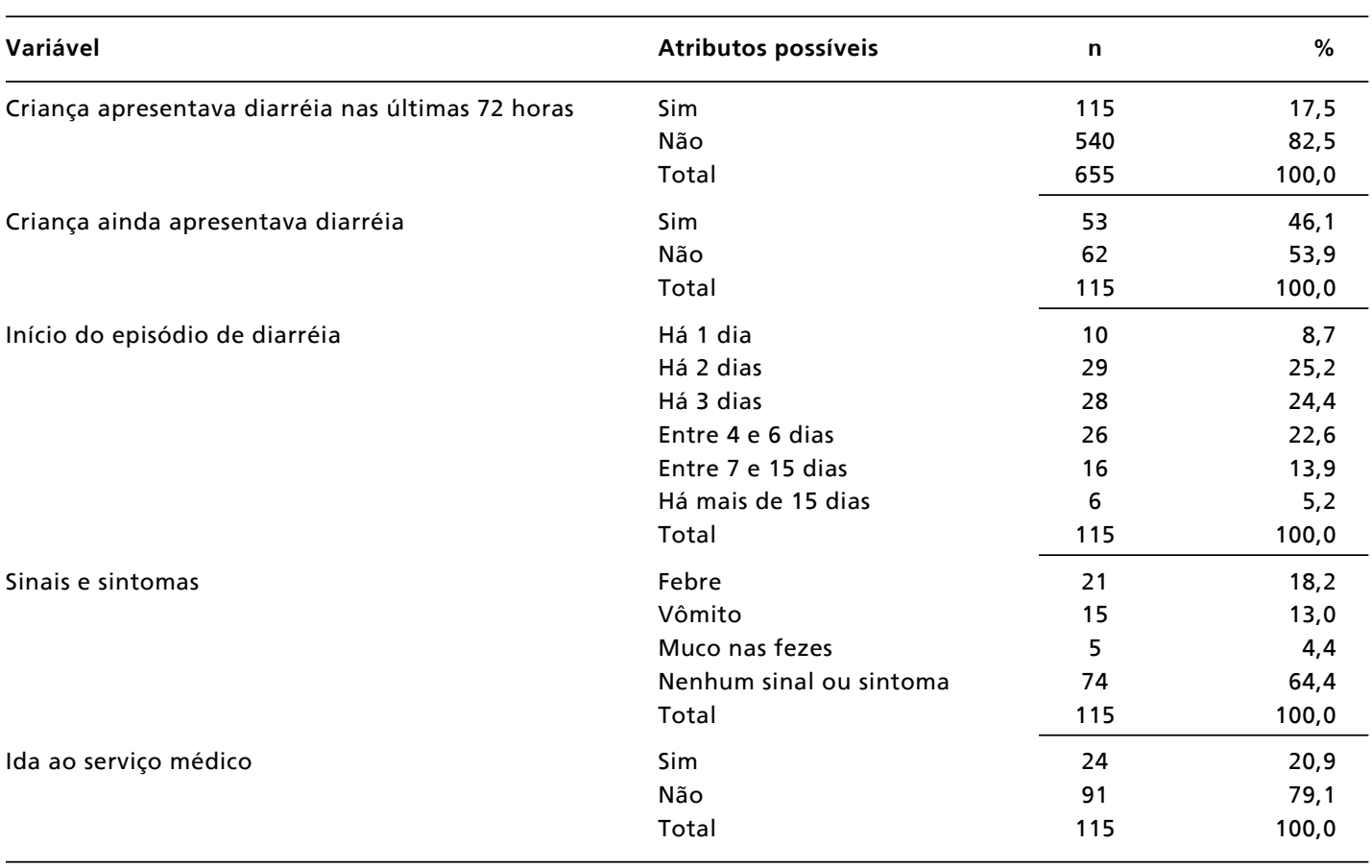

\section{Tabela 2}

Fatores associados à diarréia - análise multivariada - Odds Ratio (OR) e intervalo de confiança a 95\% (IC95\%).

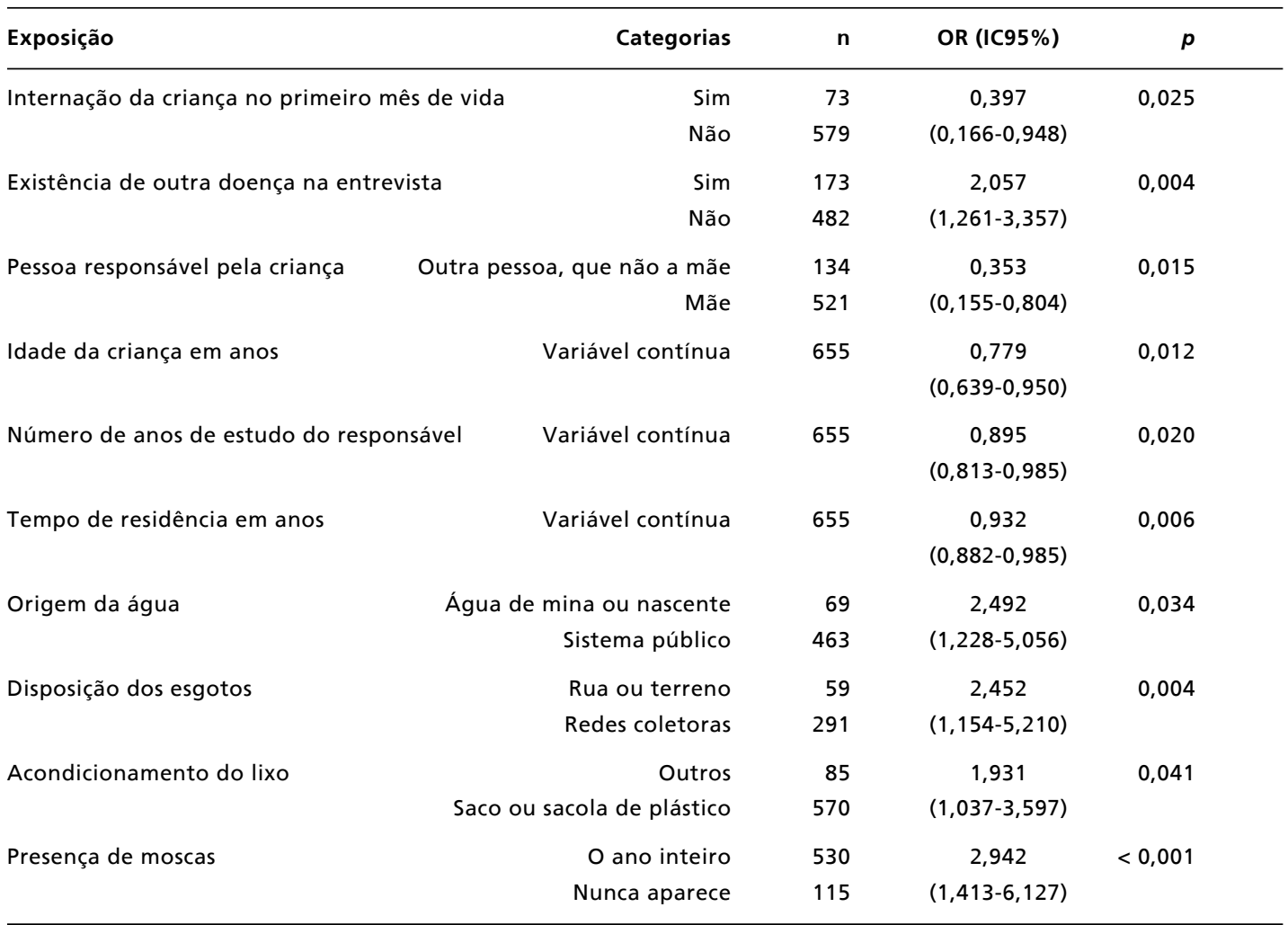




\section{Discussão}

A necessidade de internação da criança no primeiro mês de vida apresentou um caráter de proteção com OR de 0,397 (0,166-0,948). Como explicação para esse resultado, deve-se destacar que as crianças internadas no primeiro mês de vida e que sobrevivem têm maior acesso aos serviços de saúde públicos, uma vez que as mães são orientadas a procurar as Unidades Básicas de Saúde, onde é desenvolvido o Programa de Atenção ao RecémNascido de Risco, com garantia de consultas periódicas com pediatras. Tal cuidado, na maioria das vezes, não é garantido às demais crianças nos assentamentos subnormais.

A existência de doença diferente da diarréia na criança, no dia da entrevista, apresentou um odds ratio de 2,057 (1,261-3,357). Portanto, a existência de outra doença está estatisticamente associada à diarréia. O resultado era esperado, uma vez que a diarréia constitui sinal de diversas doenças. 8,9 As doenças mais citadas pelos entrevistados como enfermidades que atingiam as crianças no dia da entrevista foram, pela ordem: desnutrição, infecções respiratórias agudas e doenças infecciosas (caxumba, catapora, rubéola, etc).

A pessoa que ficou mais tempo tomando conta da criança nos últimos 12 meses comparando uma terceira pessoa (pai, professora da creche, avó, tia, etc. - que não a mãe) com a mãe apresentou caráter de proteção com OR de 0,353 (0,155-0,804). Tal resultado, em princípio, contraria o que é registrado pela literatura. Como explicar este aparente paradoxo? Ou tal resultado seria esperado?

A hipótese sustentada é de que o resultado é coerente, uma vez que a maioria dos pais que não cuida de suas crianças nos assentamentos subnormais, os deixam em creches comunitárias, onde a criança fica de 6:30 às 18:00 horas. Nessas creches, a criança tem quatro refeições diárias, banho, acompanhamento semanal por parte de pediatra, saneamento ambiental adequado e atividades recreativas. Nas áreas de assentamento subnormal, as crianças que ficam sob a guarda das mães, muitas vezes não têm assegurada as refeições diárias, ficando expostas às condições de saneamento deficientes, práticas de higiene precárias, além da dificuldade de acesso ao serviço público de saúde. Tal hipótese se sustenta na luta contínua, das famílias residentes nos assentamentos subnormais, visando ao aumento do número de vagas disponíveis nessas instituições, por parte do poder público.

A idade da criança apresentou um odds ratio de $0,779(0,639-0,950)$. Ou seja, quanto maior a criança, menor a probabilidade de apresentar diarréia. Vasta documentação científica confirma a existência de associação entre diarréia e baixa idade das crianças, 10-12 sustentada, sobretudo, na explicação de que crianças mais velhas apresentam sistema imunológico mais desenvolvido e, portanto, maior resistência a fatores etiológicos da diarréia.

O número de anos de estudo do responsável pela criança apresentou caráter protetor, o que é coerente com pesquisas anteriores. 4,10

Tempo de residência apresentou um odds ratio de 0,932 (0,882-0,985). Tal resultado era esperado, uma vez que, segundo Heller et al. ${ }^{13}$ e Moraes, ${ }^{4}$ quanto maior o tempo de residência da família na moradia, maior o investimento dos moradores na melhoria de suas condições de habitabilidade, com destaque, em particular, para os investimentos em melhoria das instalações sanitárias domiciliares.

Quanto à origem da água, o consumo de água de mina ou de nascente indicou risco significativo para diarréia - 2,5 vezes maior que o fornecimento de água pelo sistema público. Esperava-se, realmente, que as populações atendidas pela rede de abastecimento de água sob a responsabilidade da Companhia de Saneamento Municipal, estivessem com a saúde mais protegida se comparadas com aquelas atendidas por outras fontes de abastecimento de água.

O valor do odds ratio encontrado, que corresponde a uma redução de $60 \%$ na diarréia para uma água de qualidade adequada, traz novos elementos ao conhecimento na área. Esrey et al.,14 após a revisão de 144 estudos publicados em todo o mundo, concluíram que melhorias no abastecimento de água, a saber, na qualidade e na quantidade de água, levaram a uma redução de $17 \%$ na morbidade por diarréia, para os estudos mais rigorosos. Azevedo 15 detectou benefícios significativos (OR = 1,92; redução: 48\%) para a prevenção da diarréia infantil comparando um grupo que dispunha de água do sistema público de abastecimento, com outro, que não dispunha desse serviço.

A disposição dos esgotos na rua ou no terreno apresentou caráter de risco para a diarréia $(\mathrm{OR}=$ 2,45; redução: 59\%). Em relação a essa associação, Esrey et al. ${ }^{14}$ identificaram uma redução mediana de $36 \%$ na morbidade por diarréia, quando da implantação de redes coletoras de esgotos. Gross et al., 16 em um estudo longitudinal realizado em duas áreas de as-sentamento subnormal de Belo Horizonte, envolvendo crianças menores de seis anos, mostraram que a implantação de sistemas de esgotamento sanitário reduziu a incidência de diarréia em mais de $40 \%$. 
Quanto ao risco associado ao acondicionamento inadequado do lixo e a prevalência de diarréia, começa a haver maior consistência científica na literatura. Heller et al., 13 encontraram um risco relativo de 1,97 (redução: 49\%) para essa associação. Moraes 17 afirmou que, quanto à incidência de diarréia, as crianças menores de cinco anos residentes em logradouros com coleta e acondicionamento regular e irregular de lixo experimentaram, respectivamente, $65 \%$ e $43 \%$ menos episódios de diarréia do que aquelas que residiam em locais sem coleta e sem acondicionamento de lixo. Tais achados reforçam a tese defendida por Cairncross et al. 18 de que, havendo dois importantes domínios na transmissão das doenças - público e privado - a intervenção em ambos é essencial, sendo que o caso específico do acondicionamento inadequado de lixo exige intervenções no domínio privado, ou seja, no interior das moradias.

Por último, foi verificada nessa pesquisa a existência de uma associação de risco entre a presença de moscas - o ano inteiro na moradia versus nunca aparecem - e a prevalência de diarréia infantil. Tal achado confirma a associação entre a presença de moscas e diarréia verificada em outros estudos, 19,20 corroborando o importante papel desempenhado pelas moscas como vetores de doenças, destacando-se aqui, a diarréia.

\section{Referências}

1. Black RE, Morris SS, Bryce J. Where and why are 10 million children dying every year? Lancet 2003; 361: 222634.

2. Benicio MHD'A, César CLG, Gouveia MC. Perfil de morbidade e padrão de utilização de serviços de saúde das crianças brasileiras menores de cinco anos 1989. In: Monteiro MFC, Cervini R, organizadores. Perfil estatístico de crianças e mães no Brasil. Aspectos de saúde e nutrição de crianças no Brasil 1989. Rio de Janeiro: IBGE, Unicef; 1992. p. 79-96.

3. Coetzer PWW, Kroukamp LM. Diarrhoeal disease epidemiology and intervention. S Afr Med J 1989; 76: 46572.

4. Moraes LRS. Avaliação do impacto sobre a saúde das ações de saneamento ambiental em áreas pauperizadas de Salvador - Projeto AISAM. In: Heller L, Moraes LRS, Monteiro TCN, Salles MJ, Almeida LM, Câncio J, organizadores. Saneamento e saúde em países em desenvolvimento. Rio de Janeiro: CC\& P; 1997. p. 281-305.

5. Juiz de Fora (Minas Gerais). Prefeitura. JF Bairros. Juiz de Fora; 2001.

6. IBGE (Fundação Instituto Brasileiro de Geografia e Estatística). Censo demográfico: resultado do universo relativo às características da população e do domicílio. Rio de Janeiro; 1991.
A elevada prevalência de diarréia infantil encontrada em assentamentos subnormais de uma cidade de porte médio do Sudeste brasileiro $(17,5 \%)$, comparável à encontrada na região Nordeste do Brasil, leva à conclusão de que a doença concentrase nas periferias das cidades de grande e médio porte brasileiras e nas regiões economicamente mais pobres do país. Para o combate à diarréia infantil sugere-se atenção especial à saúde e à alimentação de crianças com idade abaixo de cinco anos e cujos responsáveis tenham menor nível de escolaridade, incluindo, ainda, as famílias com menor tempo de residência nos assentamentos. O estudo indica que, para o controle da diarréia, são imprescindíveis adequadas cobertura e qualidade nos serviços de saneamento ambiental. Programas de educação sanitária também se revelam fundamentais. Por fim, enfatiza-se que um ambiente saudável é pré-requisito indiscutível para a saúde e o papel do saneamento na conquista dessa condição constitui consenso.

\section{Agradecimentos}

Ao Conselho Nacional de Desenvolvimento Científico e Tecnológico (CNPq), Processo no 476.900/2001-8, pelo suporte financeiro.
7. Moraes LRS. Impacto na saúde de ações integradas de saneamento ambiental em assentamento humano periurbano de Salvador. In: Heller L, Moraes LRS, Monteiro TCN, Salles MJ, Almeida LM, Câncio J, organizadores. Saneamento e saúde em países em desenvolvimento. Rio de Janeiro: CC \& P; 1997. p. 355 [resumo].

8. Correia LL, McAuliffe JF. Saúde materno-infantil. In: Rouquayrol MZ, Almeida Filho N, editores. Epidemiologia e saúde. 5. ed. Rio de Janeiro: MEDSI; 1999. p. 375-403.

9. Lima AJ, Tortoni C. Diarréia. In: Lima AJ, editor. Pediatria essencial. São Paulo: Atheneu; 1998. p. 415-21.

10. Fuchs SC, Victora CG. Risk and prognostic factors for diarrheal disease in Brazilian infants: a special case-control design application. Cad Saúde Pública 2002; 18: 773-82.

11. Fuchs SC, Victora CG, Fachel J. Modelo hierarquizado: uma proposta de modelagem aplicada à investigação de fatores de risco para diarréia grave. Rev Saúde Pública 1996; 30: 168-78.

12. Vázquez ML, Mosquera M, Cuevas LE, González ES, Veras ICL, Luz EO, Batista Filho M, Gurgel RQ. Incidência e fatores de risco de diarréia e infecções respiratórias agudas em comunidades urbanas de Pernambuco, Brasil. Cad Saúde Pública 1999; 15: 163-72. 
13. Heller L, Colosimo EA, Antunes CMF. Environmental sanitation conditions and health impact: a case control study. Rev Soc Bras Med Trop 2003; 36: 41-50.

14. Esrey SA, Potash JB, Roberts L, Shiff C. Health benefits from improvements in water supply and sanitation: survey and analysis of the literature on selected diseases. Arlington, VA: Water and Sanitation for Health Project; 1990. (WASH Technical Report No. 66.)

15. Azevedo EA. Exclusão sanitária em Belo Horizonte - MG: caracterização e associação com indicadores de saúde [dissertação mestrado]. Belo Horizonte: Escola de Engenharia da Universidade Federal de Minas Gerais; 2003

16. Gross R, Schell B, Bisi Molina MC, Leão MAC, Strack U. The impact of improvement of water supply and sanitation facilities on diarrhea and intestinal parasites: a Brazilian experience with children in two low-income urban communities. Rev Saúde Pública 1989; 23: 214-20.
17. Moraes LRS. Avaliação epidemiológica relacionada aos resíduos sólidos domiciliares urbanos: um estudo de caso. In: Anais do XIX Congresso Brasileiro de Engenharia Sanitária e Ambiental; 1997 set., 19-23; Rio de Janeiro, Brasil. Rio de Janeiro, ABES; 1997. p. 1643-9.

18. Caincross S, Blumenthal U, Kolsky P, Moraes LRS, Tayeh A. The public and domestic domains in the transmission of disease. Trop Med Int Health 1996; 1: 27-34.

19. U KM, Khin M, Wai NN, Hman NW, Myint TT, Butler T. Risk factors for the development of persistent diarrhea and malnutricion in Brumese children. Int J Epidemiol 1992; 21: 1021-29.

20. Vathanophas $\mathrm{K}$, Indrasukhsri $\mathrm{T}$, Bunyaratabandhu $\mathrm{P}$, Suthienkul O, Varavithya U. The study of socioeconomic, behavioural and environmental factors related to diarrhoeal diseases in children under five in congested areas of Bangkok Metropolis. J Med Assoc Thailand 1986; 69: 15662.

Recebido em 15 de maio de 2005

Versão final apresentada em 7 de outubro de 2005

Aprovado em 3 de novembro de 2005 